\title{
L'épidémiologie de la cryptosporidiose en Ontario, au Canada, suite à l'adoption du test de PCR en 2018
}

\author{
Karen Johnson ${ }^{1}$, Palvasha Thayani ${ }^{2}$, Bryna Warshawsky ${ }^{1,3}$, Yvonne Whitfield $^{1}$, Jennifer Pritchard ${ }^{1}$, \\ Michelle Murti ${ }^{1,4 *}$
}

\section{Résumé}

Contexte : La cryptosporidiose est une maladie à déclaration obligatoire en Ontario, au Canada. La détection par réaction en chaîne de la polymérase (PCR) a été introduite par un grand laboratoire communautaire en août 2017, et en 2018, la fréquence de la cryptosporidiose signalée a doublé par rapport à 2012 à 2016.

Méthodes : Nous avons évalué les cas signalés en 2018 afin de déterminer les changements épidémiologiques

Résultats : Aucun foyer d'éclosion n'a été identifié en 2018 , et $48 \%$ des cas ont été détectés par PCR, ce qui laisse entendre que l'augmentation observée s'explique probablement par la plus grande sensibilité de la PCR. De la période précédant l'adoption de la PCR à celle la suivant, la proportion de cas signalés chez les femmes a augmenté de manière significative, principalement en raison des cas diagnostiqués par PCR. Une augmentation significative de l'âge moyen a également été observée parmi les cas diagnostiqués par microscopie ou par PCR dans la période suivant l'adoption de la PCR.

Conclusion : Nos conclusions soulignent l'importance d'évaluer les méthodes diagnostiques lors de l'évaluation de l'évolution des taux signalés. L'évolution de l'incidence observée exigera une surveillance continue et pourrait nécessiter des périodes de référence plus courtes pour la détection des aberrations.
Cette oeuvre est mise à la disposition selon les termes de la licence internationale Creative Commons Attribution 4.0

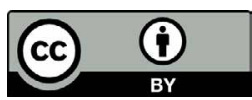

Affiliations

${ }^{1}$ Santé publique Ontario, Toronto, ON

2 École de santé publique et des systèmes de santé, Université de Waterloo, Waterloo, ON

${ }^{3}$ École de médecine et de dentisterie Schulich, Université Western, London, ON

${ }^{4}$ École de santé publique Dalla Lana, Université de Toronto, Toronto, ON

${ }^{\star}$ Correspondance : michelle.murti@oahpp.ca

Citation proposée : Johnson KO, Thayani P, Warshawsky B, Whitfield Y, Pritchard J, Murti M. L'épidémiologie de la cryptosporidiose en Ontario, au Canada, suite à l'adoption du test de PCR en 2018. Relevé des maladies transmissibles au Canada 2020;46(7/8):255-8. https://doi.org/10.14745/ccdr.v46i78a02f

Mots-clés : Cryptosporidium, cryptosporidiose, surveillance, PCR, réaction en chaîne de la polymérase, épidémiologie, détection des épidémies

\section{Introduction}

La cryptosporidiose est une maladie diarrhéique transmise par contact oro-fécal avec des personnes ou des animaux infectés. Bien que le mode de transmission varie, l'infection est surtout acquise par l'ingestion d'eau potable ou d'eaux récréatives qui a été contaminée par des oocystes de Cryptosporidium (1). La cryptosporidiose est habituellement diagnostiquée par I'examen microscopique des échantillons de selles ou par des épreuves immunoenzymatiques pour la détection des antigènes d'espèces de Cryptosporidium. Cependant, les méthodes moléculaires, y compris la réaction en chaîne de la polymérase (PCR), sont de plus en plus utilisées $(2,3)$. En Ontario, au Canada, les laboratoires des hôpitaux, le laboratoire provincial de santé publique et les laboratoires communautaires privés effectuent des tests diagnostiques pour la cryptosporidiose. Les laboratoires communautaires privés diagnostiquent la majorité des cas signalés. Tous les cas de cryptosporidiose confirmés en laboratoire doivent être déclarés en Ontario (4), et les cas signalés sont suivis par les conseils de santé locaux, qui recueillent et communiquent les renseignements sur les cas de manière centralisée aux responsables de la santé publique de la province, par l'entremise du Système intégré d'information sur la santé publique (SIISP).

La microscopie a été la principale méthode de détection de la cryptosporidiose en Ontario. En août 2017, un grand laboratoire communautaire possédant des centres de collecte dans toute 
la province a adopté le test multiplex de PCR (5). Des études récentes ont montré que la sensibilité de la microscopie se situe entre $52 \%$ et $56 \%$ par rapport à la PCR, dont la sensibilité est de $100 \%(6,7)$.

Dans cet article, nous décrivons notre analyse de l'augmentation des cas de cryptosporidiose signalés en 2018 dans le but d'évaluer l'ampleur de cette augmentation, de déterminer le rôle de la PCR dans cette augmentation et de déterminer si des changements se sont produits dans l'épidémiologie de la cryptosporidiose depuis la mise en place de la PCR.

\section{Méthodes}

Nous avons mené cette évaluation en comparant les données démographiques, les facteurs de risque et les résultats du SIISP de 2018 (la première année complète suivant la mise en œuvre du test de PCR) par rapport à une période précédant l'adoption de la PCR, soit de 2012 à 2016.

Nous avons extrait des renseignements sur l'âge, le sexe, l'issue (hospitalisation ou décès), les symptômes signalés, le laboratoire (c.-à-d. communautaire, hospitalier ou de santé publique) et la méthode diagnostique [c.-à-d. PCR, microscopie, épreuve immunoenzymatique (EIA) et culture bactérienne] du SIISP pour les cas de cryptosporidiose dont les dates d'épisodes vont du 1er janvier 2012 au 31 décembre 2016 (préalable à l'adoption de

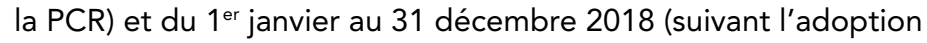
de la PCR).

Les cas sans données diagnostiques, les cas diagnostiqués par PCR au cours de la période précédant l'adoption de la PCR et les cas dont la méthode de détection était «culture-bactérienne " (ce qui tient compte des erreurs de saisie des données) ont été exclus de toutes les analyses, à l'exception du nombre total de cas et des taux. Les facteurs de risque sociaux ou comportementaux, notamment les antécédents de voyage à l'extérieur de la province, les expositions d'origine alimentaire et hydrique, et les contacts avec des animaux et entre personnes, pour les cas dont la date d'épisode se situe en 2018 , ont également été analysés pour déterminer s'il y avait eu des éclosions non reconnues auparavant. Toutes les analyses ont été effectuées à l'aide de la version 7.1 du progiciel statistique SAS Enterprise Guide ( $p<0.05$ étant considéré statistiquement significatif).

\section{Résultats}

Les cas de cryptosporidiose signalés ont augmenté chaque année, passant de 299 en 2012 à 429 en 2016, puis sont tombés à 391 en 2017 et sont passés à 751 en 2018 (figure 1). Le taux annuel de cryptosporidiose en Ontario était de 2,6 pour 100000 habitants de 2012 à 2016 et de 5,2 pour
Figure 1 : Nombre et taux d'incidence de tous les cas confirmés de cryptosporidiose signalés, en Ontario, au Canada, de 2012 à $2018(n=2$ 928)

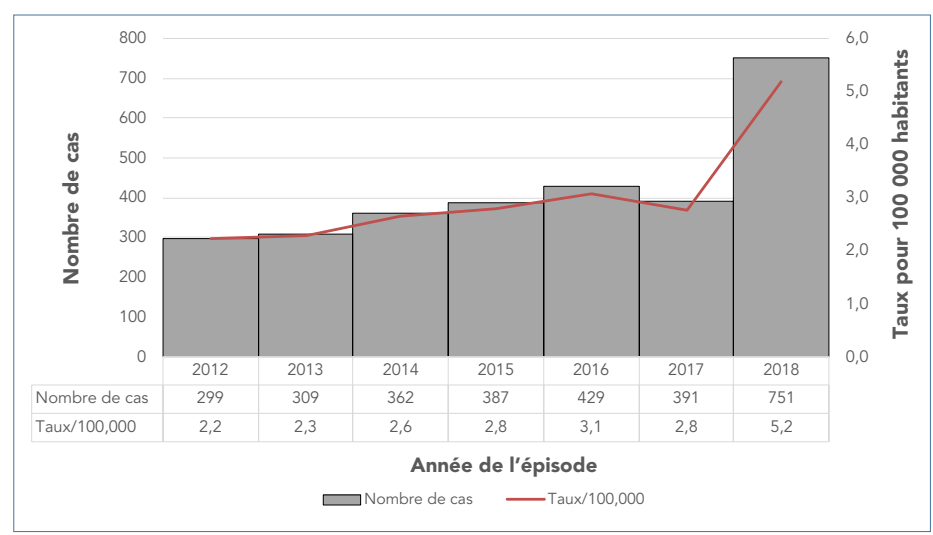

a Estimations démographiques de 1986 à 2017 and projections démographiques 2017 à 2041 ministère de la Santé de l'Ontario, SIISP Ontario, consulté le 26 novembre 2019

100000 habitants en $2018(p<0,001)$. Selon les 1743 cas de l'analyse plus détaillée, la détection par PCR représentait $48 \%$ des cas de cryptosporidiose signalés en 2018 , tandis que la détection par microscopie a diminué de $99,8 \%$ de 2012 à 2016 à $52 \%$ en $2018(p<0.001)$; l'EIA a été la méthode utilisée pour détecter trois cas signalés pendant la période précédant l'adoption de la PCR $(0,2 \%)$.

Des augmentations statistiquement significatives de l'âge moyen global et par sexe ont également été observées; les augmentations au cours de la période suivant l'adoption de la PCR se sont produites parmi les cas diagnostiqués par microscopie et par PCR (tableau 1). Dans l'ensemble, la proportion de cas chez les femmes est passée de $48,5 \%$ dans la période précédant l'adoption de la PCR à $55,4 \%$ dans la période suivant l'adoption de la PCR $(p<0,009)$. La stratification de la période suivant l'adoption de la PCR a montré que ce changement de proportion par rapport à la période précédant l'adoption de la PCR était dû aux cas diagnostiqués par PCR (60,8 \% dans la période suivant l'adoption de la PCR, $p<0,001)$, car la proportion de cas signalés chez les femmes diagnostiqués par microscopie au cours la période suivant l'adoption de la $\operatorname{PCR}(50,4 \%)$ ne variait pas de manière significative par rapport à la période précédant l'adoption de la PCR $(48,5 \%, p<0,580)$ (tableau 1).

De la période précédant et suivant l'adoption de la PCR, la proportion de cas hospitalisés a diminué de $5,5 \%$ à $2,1 \%$ $(p<0,002)$. Aucun décès n'a été enregistré au cours de ces deux périodes. La méthode de test n'était pas liée à l'état des symptômes, bien que la proportion de cas asymptomatiques ait été légèrement plus élevée parmi ceux diagnostiqués par PCR $(2,2 \%)$ et par microscopie $(1,3 \%)$ au cours de la période suivant l'adoption de la PCR par rapport à la période précédant l'adoption de la PCR (0,8 \%). 
Tableau 1 : Cas de cryptosporidiose signalés pendant la période précédant l'adoption de la PCR (2012 à 2016) par rapport aux cas signalés en 2018, dans l'ensemble et stratifiés par méthode de diagnostic $(n=1743)^{a}$

\begin{tabular}{|c|c|c|c|c|c|c|c|c|c|c|c|}
\hline \multirow{3}{*}{$\begin{array}{l}\text { Caractéristiques } \\
\text { du cas }\end{array}$} & \multicolumn{2}{|c|}{$\begin{array}{c}\text { Période } \\
\text { précédant } \\
\text { I'adoption de la } \\
\text { PCR } \\
\text { (2012 à 2016) }\end{array}$} & \multicolumn{9}{|c|}{$\begin{array}{l}\text { Période suivant l'adoption de la PCR } \\
\qquad(2018)^{b}\end{array}$} \\
\hline & \multirow{2}{*}{\multicolumn{2}{|c|}{$\begin{array}{l}\text { Microscopie } \\
(n=1209) \text { et } \\
\text { EIA }(n=3)\end{array}$}} & \multicolumn{3}{|c|}{ PCR } & \multicolumn{3}{|c|}{ Microscopie } & \multicolumn{3}{|c|}{ Microscopie et PCR } \\
\hline & & & \multicolumn{2}{|c|}{$n=255$} & valeur $p^{c}$ & \multicolumn{2}{|c|}{$n=276$} & valeur $p^{c}$ & \multicolumn{2}{|c|}{$n=531$} & valeur $p^{c}$ \\
\hline Diagnostiqué (\%) & \multicolumn{2}{|c|}{$\begin{array}{c}\text { Microscopie } \\
(99,8 \%), \operatorname{EIA}(0,2 \%)\end{array}$} & \multicolumn{2}{|r|}{$48,0 \%$} & - & \multicolumn{2}{|r|}{$52 \%$} & - & \multicolumn{2}{|r|}{$100 \%$} & - \\
\hline \multicolumn{12}{|l|}{ Âge moyen (années) } \\
\hline Femme & \multicolumn{2}{|r|}{23,3} & \multicolumn{2}{|r|}{27,3} & $<0,005$ & \multicolumn{2}{|r|}{28,4} & $<0,001$ & \multicolumn{2}{|r|}{27,8} & $<0,001$ \\
\hline Homme & \multicolumn{2}{|r|}{20,9} & \multicolumn{2}{|r|}{26,4} & $<0,002$ & \multicolumn{2}{|r|}{24,9} & $<0,011$ & \multicolumn{2}{|r|}{25,5} & $<0,001$ \\
\hline Total $^{d}$ & \multicolumn{2}{|r|}{22,1} & \multicolumn{2}{|r|}{26,9} & $<0,001$ & \multicolumn{2}{|r|}{26,6} & $<0,001$ & \multicolumn{2}{|r|}{26,8} & $<0,001$ \\
\hline \multicolumn{12}{|l|}{ Sexe, n (\%) ${ }^{d}$} \\
\hline Femme & 588 & $(48,5)$ & 155 & $(60,8)$ & $<0,001$ & 139 & $(50,4)$ & $<0,580$ & 294 & $(55,4)$ & $<0,009$ \\
\hline Homme & 620 & $(51,2)$ & 100 & $(39,2)$ & $<0,001$ & 136 & $(49,3)$ & $<0,573$ & 236 & $(44,4)$ & $<0,010$ \\
\hline Hospitalisations, n (\%) & 67 & $(5,5)$ & 4 & $(1,6)$ & $<0,008$ & 7 & $(2,5)$ & $<0,040$ & 11 & $(2,1)$ & $<0,001$ \\
\hline \multicolumn{12}{|l|}{ État des symptômes ${ }^{\mathrm{e}}$} \\
\hline $\begin{array}{l}\text { Asymptomatique, } \\
\mathrm{n}(\%)\end{array}$ & 9 & $(0,8)$ & 5 & $(2,2)$ & $<0,084$ & 3 & $(1,3)$ & $<0,461$ & 8 & $(1,7)$ & $<0,129$ \\
\hline \multicolumn{12}{|c|}{ 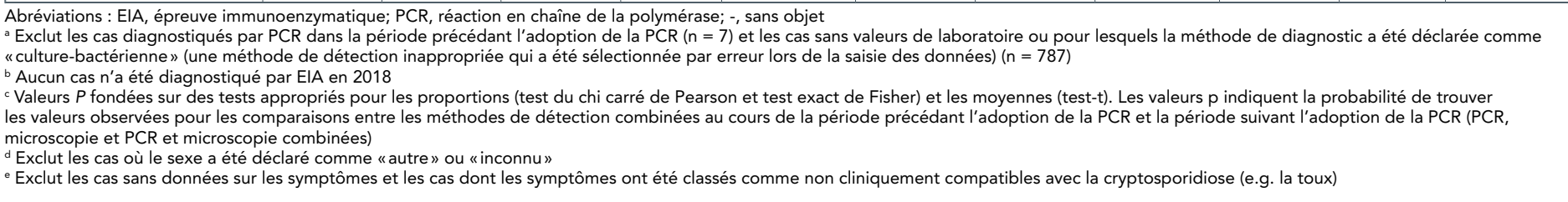 } \\
\hline
\end{tabular}

Selon les facteurs de risque évalués, aucun point commun n'a été signalé à l'échelle provinciale qui pourrait suggérer l'apparition d'éclosions parmi les cas non liés aux voyages en 2018, ou au sein des unités sanitaires qui avaient observé des augmentations localisées au-delà de leur pic d'incidence habituel de l'été.

\section{Conclusion}

Notre enquête a révélé que l'incidence des cas de cryptosporidiose avait doublé en 2018, première année complète après l'adoption de la PCR en août 2017, par rapport à la période précédant l'adoption de la PCR, soit de 2012 à 2016. Cependant, on ne sait pas encore très bien pourquoi le nombre de cas signalés en 2017 a diminué malgré l'adoption de la PCR cette année-là et une tendance à l'augmentation progressive au cours des cinq années précédentes. Comme seuls les résultats de laboratoire positifs doivent être signalés en Ontario, nous n'avons pas pu déterminer si l'augmentation en 2018 était due à une augmentation du nombre de cas, à une augmentation du nombre de tests commandés ou à une augmentation de la positivité en raison de la plus grande sensibilité de la PCR. Notre enquête sur l'augmentation en 2018 n'a pas non plus identifié de facteurs de risque sociaux et comportementaux communs parmi les cas au pays qui étaient indicatifs d'une éclosion. Pendant la période d'augmentation, les indications cliniques visant les tests et les critères de surveillance pour définir et signaler les cas n'ont pas changé, et les prestataires de soins de santé n'ont pas eu la possibilité de préciser leur méthode de diagnostic privilégiée (e.g. microscopie, EIA ou PCR) lorsqu'ils ont commandé des tests. Compte tenu de ces facteurs, il est possible que l'augmentation en 2018 soit issue, du moins partiellement, de la plus grande sensibilité de la PCR, qui a permis de détecter des cas qui, autrement, seraient restés non diagnostiqués par microscopie ou EIA.

Avec l'augmentation du taux de cryptosporidiose signalé, on a également constaté une augmentation de la proportion de cas chez les femmes en 2018, en raison des diagnostics par PCR. Une hypothèse possible est que le taux de positivité du test de base chez les femmes était plus faible que chez les hommes et qu'il était donc plus affecté par la plus grande sensibilité du test de PCR. En revanche, l'augmentation de l'âge moyen des cas diagnostiqués à la fois par PCR et par microscopie en 2018 pourrait refléter un changement sous-jacent dans la répartition par âge des personnes testées en 2018 par rapport à la période de 2012 à 2016. Nous aurions besoin de données sur l'âge et le sexe des personnes qui ont soumis des échantillons pour les 
tests, les volumes de tests et les taux de positivité pour évaluer pleinement ces changements démographiques associés aux cas de cryptosporidiose.

La tendance à la baisse des hospitalisation et l'augmentation des cas asymptomatiques après l'adoption du test de PCR traduit la plus grande sensibilité et l'utilisation de la PCR dans les laboratoires communautaires qui testent les patients non hospitalisés. Des tendances semblables parmi les cas détectés au microscope ont également été observées, ce qui suggère que d'autres facteurs que l'adoption du test de PCR pourraient avoir influencé les changements de statut d'hospitalisation et de symptômes en 2018. Cependant, étant donné la faible proportion de cas globalement hospitalisés ou asymptomatiques, il faut faire preuve de prudence avant de tirer des conclusions à partir des données limitées sur les cas suivant l'adoption de la PCR.

Notre enquête a démontré que l'approche traditionnelle d'examen de l'incidence au fil du temps des tendances et de la détection des éclosions doit être couplée à un examen d'autres éléments de données tels que les méthodes de diagnostic et les renseignements sur les facteurs de risque, qui peuvent indiquer si les augmentations sont des artéfacts, dues à des augmentations de cas sporadiques ou attribuables à une source spécifique. Nous prévoyons que l'adoption du test de PCR pour le diagnostic de la cryptosporidiose se fera à des moments différents par les différents laboratoires de diagnostic de l'Ontario, ce qui entraînera des augmentations échelonnées de l'incidence. Ces changements exigeront une réévaluation continue des valeurs de référence pour une interprétation précise des tendances en vue de la détection des aberrations et de la détection rapide des éclosions. Avec le temps, nous nous attendons à l'émergence d'une base de référence plus stable et plus durable, avec un taux d'incidence plus élevé en raison de l'utilisation plus répandue de la PCR. Comme notre enquête reflète une année de test PCR, l'analyse d'années supplémentaires de données est nécessaire pour comprendre la pertinence clinique et de santé publique des cas détectés par cette méthode.

\section{Déclaration des auteurs}

K. J. - Analyse des données, rédaction - révision et correction P. T. - Analyse des données, rédaction - révision et correction B. W. - Révision des données, rédaction - révision et correction

Y. W. - Révision des données, rédaction - révision et correction J. P. - Administration du projet, rédaction — révision et correction

M. M. - Rédaction - version initiale du projet, rédaction révision et correction

\section{Intérêts concurrents}

Aucun.

\section{Financement}

Cet article a reçu l'appui de Santé publique Ontario.

\section{Remerciements}

Nous remercions L. Friedman, de Santé publique Ontario, qui a assuré la révision des textes et la gestion des références.

\section{Références}

1. Heymann DL, editor. Control of communicable diseases manual. 19th ed. Washington (DC): American Public Health Association; 2008.

2. van Lieshout $L$, Roestenberg M. Clinical consequences of new diagnostic tools for intestinal parasites. Clin Microbiol Infect 2015;21(6):520-8. DOI PubMed

3. Adeyemo FE, Singh G, Reddy P, Stenström TA. Methods for the detection of Cryptosporidium and Giardia: from microscopy to nucleic acid based tools in clinical and environmental regimes. Acta Trop 2018;184:15-28. DOI PubMed

4. Ministry of Health and Long-term Care. Infectious diseases protocol: Appendix B: Provincial case definitions for diseases of public health significance; Disease: Cryptosporidiosis. Toronto (ON): Government of Ontario; 2019. http://www. health.gov.on.ca/en/pro/programs/publichealth/oph_ standards/docs/crypto_cd.pdf

5. LifeLabs. Client notice update - LifeLabs introduces PCR testing for detection of parasites in stool samples. Toronto (ON): LifeLabs; 2017 Aug 24 (accédé 2018-07-26). https:// www.lifelabs.com/client-notice-update-lifelabs-introduces -pcr-testing-for-detection-of-parasites-in-stool-samples/

6. Stark D, Al-Qassab SE, Barratt JL, Stanley K, Roberts T, Marriott D, Harkness J, Ellis JT. Evaluation of multiplex tandem real-time PCR for detection of Cryptosporidium spp., Dientamoeba fragilis, Entamoeba histolytica, and Giardia intestinalis in clinical stool samples. J Clin Microbiol 2011;49(1):257-62. DOI PubMed

7. Laude A, Valot S, Desoubeaux G, Argy N, Nourrisson C, Pomares C, Machouart M, Le Govic Y, Dalle F, Botterel F, Bourgeois N, Cateau E, Leterrier M, Le Pape P, Morio F. Is real-time PCR-based diagnosis similar in performance to routine parasitological examination for the identification of Giardia intestinalis, Cryptosporidium parvum/ Cryptosporidium hominis and Entamoeba histolytica from stool samples? Evaluation of a new commercial multiplex PCR assay and literature review. Clin Microbiol Infect 2016;22(2):190.e1-8. DOI PubMed 